\title{
Competency and use of the Mental Health Act - a matrix to aid decision-making
}

\author{
AIMS AND METHOD \\ Competence in patients with mental \\ illness is an issue of growing impor- \\ tance. We present a matrix that pro- \\ vides a new tool to separate the \\ elements involved.
}

\author{
RESULTS \\ This matrix is compatible with, and \\ makes explicit, the Richardson \\ Committee's suggestions concerning \\ the incorporation of a test of capacity \\ in Mental Health Act assessments.
}

\begin{abstract}
CLINICAL IMPLICATIONS
We suggest that use of this matrix may offer practical help to clinicians in arriving at a clear understanding of each clinical situation and guide good practice in the use of the Mental Health Act, particularly in problematic cases.
\end{abstract}

\section{Concepts of competence}

Common Law in England and Wales gives patients the right to refuse treatments that are offered to them, as long as they possess the capacity to make these choices (ReC, 1994). It is this capacity, rather than other forms such as testamentary capacity, that will be referred to in this paper. The criteria of capacity to consent to, or refuse, treatment are: the ability to understand information; the ability to believe it; and the ability to weigh it in the balance in order to reach a decision ( $R e C, 1994$; British Medical Association, 1995). Competence is the clinical equivalent to the legal concept of capacity to consent to treatment. In practice, mental health professionals probably use a wider working definition of competence in patients than the strict legal criteria of capacity, and take into consideration other factors such as the patient's ability to apply the knowledge to his or her own situation, the consistency of decisions and the value systems of the patient (Grisso \& Appelbaum, 1998). Capacity and competence, however, can be different over time and situation, and any assessment of competence is only valid for the time and circumstances for which the assessment is made.

\section{Competence and the Mental Health Act}

The Mental Health Act provides a mechanism that empowers the psychiatrist to detain and treat mental illness without the patient's consent. There is currently no explicit reference to competence in the Mental Health Act assessment procedures.

It is obviously desirable from the point of view of individual rights of competent patients, to protect them from overzealous use of the Mental Health Act when they refuse treatment. The reverse danger, as illustrated by the recent Bournewood case ( $L$ v. Bournewood Community and Mental Health NHS Trust, 1997), is that patients lacking competence may not have their rights preserved if they are held without either informed consent or legal means, a situation that the Law Lords recognised would leave them with fewer avenues for advocacy or appeal (ReL, 1998).
This tension with regard to competence and the Mental Health Act was recognised in the recently published Mental Health Act Review draft proposals (Department of Health, 1999a). The Richardson Committee drawing up the proposals recommended that Mental Health Act assessments should incorporate an assessment of capacity, in order to factor into the consideration of compulsory treatment the promotion of autonomy of those possessing capacity, and the protection of the interests of those who lack it. Further, 'the approach proposed by the Committee would mean that to meet the criteria for imposition of a compulsory order, patients with capacity would have to be assessed as presenting a higher degree of risk than those without capacity.' (Department of Health, 1999b: p. 32)

The subsequent White Paper (Department of Health, 2000) does not propose specific reference to capacity in the criteria for compulsory treatment. However, it recognises that children, young people and patients with long-term mental incapacity need additional protection with respect to refusal of treatment. Furthermore, changes in society mean that it is likely that the assessment of competence will become a core clinical skill for psychiatrists, even in situations where it is more temporarily impaired by mental disorder. For,. . . as a matter of good practice the care team would always be expected to take steps to promote the patient's ability to consent to treatment and involve them in decisions on their own care. This is in line with the suggested principle of involvement and specific guidance will be included in the Code of Practice.' (Department of Health, 1999b: p. 33)

\section{Development of the competency matrix}

The authors were involved in a difficult case, which led to the development of the competency matrix. Mr D, a 34year-old patient with lifelong renal problems arising from a meningomyelocele, was on renal dialysis following the rejection of a renal transplant, and was suffering pain from the site. He became low in mood and on assessment satisfied full criteria for a depressive disorder. He was offered antidepressants but refused them as well as 
his renal dialysis, with clear intent to die. Owing to the complexity and urgency of the situation, the psychiatrists and renal physicians had difficulty resolving the situation. Based on the evidence that he was suffering from a depressive illness and was now refusing life-sustaining treatment as a method of passive suicide, Mr D was placed on Section 2 of the Mental Health Act, following the failure of persuasion to continue with renal dialysis or accept psychiatric treatment. He was given renal dialysis under sedation once as a life-saving measure under Common Law, as being possibly incompetent to refuse treatment owing to his depressive disorder, then transferred to a psychiatric hospital.

Once in a psychiatric hospital, Mr D consented to his renal dialysis and complied with his treatment, and he was rapidly taken off the compulsory order and transferred back to the renal ward. His mood lifted with treatment and he was discharged home with out-patient psychiatric follow-up.

Unfortunately, after 2 to 3 months Mr D's pain returned, his mood dropped and he again decided to refuse dialysis. He was assessed by the duty psychiatrist, who concluded that Mr D was not suffering from a mental illness within the meaning of the Mental Health Act, and had the capacity to refuse treatment. Therefore the psychiatrists and renal physicians in consultation allowed him to refuse his dialysis. Mr D was admitted to a hospice and given palliative care, and he died peacefully 6 days later.

Following this complex case, there was a sense among the physicians and psychiatrists involved that the multiple factors involved, as well as the life-threatening medical situation, had made it very difficult to think through the appropriateness of use of the Mental Health Act. The authors felt, in hindsight, that there was no clear existing framework to guide clinicians and provide consistency in the way that the various factors of the patient's mental state, the issue of competence and the Mental Health Act were considered. The use of an objective framework could have facilitated comparison in the two different occasions when he refused his renal dialysis, and been the basis of meaningful discussions between the patient, his family and the health professionals involved. This led to the authors coming up with a matrix, which aided them in thinking about this situation more clearly and consistently.

\section{A mental disorder and competency matrix}

In considering these two different issues of mental disorder and competence, a matrix can be constructed (Fig. 1). Using the matrix first directs the clinician to consider each of three questions separately and in turn. This may be its greatest value.

Patients falling in cells $2 a, 2 b, 4 a$ and $4 b$ generally fulfil criteria for use of the Mental Health Act, with some exceptions for psychopathic disorder and mental impairment. Patients falling in cells $8 \mathrm{a}$ and $8 \mathrm{~b}$ cannot be treated under the Mental Health Act, but they can, along with patients in cells $4 \mathrm{a}$ and $4 \mathrm{~b}$, be treated without consent under Common Law in emergency situations. The exact definitions of mental disorder and risk are clinical decisions, as the Mental Health Act itself makes clear. It would be a useful exercise for the reader to try to generate examples for each cell in the matrix.

\section{Using the matrix to aid decision-making}

This matrix has been piloted among some mental health professionals in the Oxford area, as well as medical ethicists. The general feedback has been that this matrix is thought-provoking and can be helpful in clarifying the factors involved, and one clinician felt it may even change the decision-making process.

When difficult clinical decisions arise that involve refusal of treatment, we suggest that the matrix can be helpful to the average clinician, who does not possess expertise in issues of competence, in thinking through the appropriateness of using the Mental Health Act. During the assessment of the situation, three factors should be looked for independently: the presence and degree of risk to self or others; the presence of mental disorder; and the competence of the patient to consent to the particular treatment being proposed, as assessed at the time of using the matrix. It must be emphasised that the thresholds for deciding what constitutes sufficient degree of risk to be classified as danger to self or danger to others, and what constitutes competence in the given situation, are purely clinical decisions. Indeed, the thresholds can and must be set according to the degree of urgency, the seriousness of consequences and the circumstances of the particular situation. For instance, if the issue is danger to young children in the sole care of a patient suffering from a serious mental disorder, then the clinicians may choose to set a lower threshold for what would constitute danger to others.

\begin{tabular}{|l|c|c|c|c|c|c|}
\hline \multicolumn{3}{|c|}{ Competent } & \multicolumn{3}{c|}{ Incompetent } \\
\hline Mental disorder & No risk & Risk to self & Risk to others & No risk & Risk to self & Risk to others \\
\hline Present & Cell 1 & Cell $2 a$ & Cell $2 b$ & Cell 3 & Cell 4a & Cell 4b \\
\hline Absent & Cell 5 & Cell $6 a$ & Cell 6b & Cell 7 & Cell 8a & Cell 8b \\
\hline
\end{tabular}

Use of matrix includes the three important questions. For criteria for use of the Mental Health Act or not see text.

Fig. 1 The competency to consent matrix 
Then, using the matrix, the cell within which the patient falls can be very easily identified in an objective fashion. If the patient falls within cells $4 a$ and $4 b$, the

special articles decision to use the Act is well established. In fact, as the patient is lacking competence, any decisions not to apply the Act should be carefully considered. However, if the patient is found to fall in cells $2 a$ or $2 b$ as being competent, the Richardson Committee (Department of Health, 1999a) suggests setting a higher standard of risk required before compulsory powers are used, so that patient autonomy is protected.

Avoidance of the use of compulsory treatment where possible is in the spirit of the Mental Health Act, even where lack of competence exists. The function of the matrix is not to prescribe its use, but rather to allow the consideration of appropriateness in a more formal framework. Breaking down the global decision into its component factors and making the steps in consideration of compulsory treatment more explicit, may be helpful in situations where there are multiple factors rendering the decision difficult.

\section{Conclusion}

The issues of competence and the use of compulsory treatment can be very difficult, with individual clinical cases presenting great challenges to clinicians. We were involved in one such difficult case, which led to the development of this matrix.

It is true that in real life these three factors in the matrix are interrelated and each affects the other, as previously discussed. It is also true that clinical decisions must be made on the basis of balancing further factors of urgency, level of risk and the seriousness of the intervention proposed. This matrix is not prescriptive about the course of action that should follow, nor of the scope of definition of the terms mental disorder, risk and competence. Instead, its role is more to aid separate consideration of the different dimensions involved in order to indicate the relevance of compulsory treatment. We would suggest that the use of a simple matrix in difficult cases may aid good practice in decision-making.

\section{References}

BRITISH MEDICAL ASSOCIATION (1995) Assessment of Mental Capacity: Guidance for Doctors and Lawyers. London: BMA.

DEPARTMENT OF HEALTH (1999a) Review of the Mental Health Act 1983 . Draft Outline Proposals by Scoping Study Committee. London: Stationery Office.

- (1999b) Reform of the Mental Health Act 1983: Proposals for

Consultation. Presented to Parliamen by the Secretary of State for Health by

Command of Her Majesty. Cm 4480. London: Stationery Office.

—(2000) Reforming the Mental Health Act. Part 1. The New Legal Framework. Presented to Parliament by the Secretary of State for Health and the Home Secretary by Command of Her Majesty. Cm 5016-I. London: Department of Health.

GRISSO,T. \& APPELBAUM, P. S. (1998) Assessing Competence to Consent to Treatment: A Guide for Physicians and other Health Professionals. Oxford: Oxford University Press.

Lv. Bournewood Community and Mental Health NHS Trust (1997) TLR 8/12/97. ReC (Adult: Refusal of Medical Treatment) (1994) 1 All ER 819. ReL (By His Next Friend GE) (1998) TLR 25/6/98

*JacintaTan Specialist Registrar, Child \& Adolescent Psychiatry, The Park Hospital for Children, Old Road, Headington, Oxford OX5 7LO

Martin Elphick Consultant Psychiatrist, The Elms Clinic, Oxford

\section{Mentoring for newly appointed consultant psychiatrists}

The south-west Mentoring Scheme has developed in the context of a general and increasing concern for the health and morale of senior doctors (British Medical Association (BMA), 2000). Their problems are now well described (Allen, 1999; Ghodse et al, 2000), but there is little evidence of effective intervention. The aspirations of the National Service Framework and the NHS Plan will come to nothing if there are not sufficient trained and experienced staff to implement them, and senior staff are increasingly looking forward to early retirement rather than the continued satisfactions of working within the NHS. Recruitment and retention of consultant psychiatrists is the most problematic of all medical specialities, with $14 \%$ of posts empty or occupied by locums (Sainsbury Centre for Mental Health, 2000). There is a clear need to find ways of preventing work-related ill health, stress and burn-out among practitioners (Roberts, 1997; Department of Health, 1999: p 115), and to sustain their creative and productive engagement in health care. A demotivated profession cannot be expected to deliver an effective service (McBride \& Metcalf, 1995; Appleton et al, 1998) and policy is not a substitute for personnel.

\section{The problems of practitioners}

Every study that has looked at the problems currently facing practitioners produces a similar long and depressing list: understaffing, poor premises, low morale, unacceptable workloads, a feeling of being dispossessed of their professional role and autonomy, violence and the fear of violence, a culture of blame, failure of management and a lack of support from colleagues (Williams et al, 1998; Ghose et al, 2000); although consultant psychiatrists work fewer hours than physicians and surgeons, they report more emotional exhaustion and depression (Deary et al, 1996). 